\section{Kommentar}

Die Studie bestätigt andere Ergebnisse, die leider noch zu wenig bekannt sind. Eine CBPT macht auch nach einer Operation Sinn (etwa von Bandscheibenvorfällen oder Lumbalkanalstenosen), auch wenn man der Ansicht ist, dass eine Operation die Ursache der Schmerzen beseitigt hat. Insbesondere bei Patienten mit einem er- höhten Risiko für das Auftreten von Schmerzen nach der Operation kann es sinnvoll sein, frühzeitig eine solche Intervention durchzuführen. Dabei sollten die Interventionen immer aus psychotherapeutischen (das heißt: behavioralen) und physiotherapeutischen Elementen kombiniert sein. Wichtig sind auch gewisse individuelle Einstellungen der Behandlung auf den Betroffenen. Insofern ist eine automatisierte Anwendung von Verhaltens-therapie nach operativen Eingriffen wahrscheinlich wenig sinnvoll.

Prof. Dr. Dr. Stefan Evers

Archer KR et al. Cognitive-behavioral-based physical therapy for patients with chronic pain undergoing lumbar spine surgery: a randomized controlled trial. J Pain 2016; 17: 76-89

\title{
Schmerz ist nicht gleich Schmerz
}

In der neuen Version der Diagnoseverschlüsselung ICD-11, die gegenwärtig von der WHO entwickelt wird, wird es erstmals ein eigenes Kapitel mit chronischen Schmerzerkrankungen geben.

$\mathrm{B}$ islang waren Schmerzerkrankungen immer unter jeweils anderen übergeordneten Kapiteln - insbesondere im Gebiet der Neurologie - aufgelistet. Nunmehr soll es ein eigenständiges Kapitel geben, das allerdings Querverweise zu anderen Kapiteln aufweist. Insgesamt werden die folgenden Schmerzgruppen genauer klassifiziert: chronischer primärer Schmerz, chronischer Tumorschmerz, chronischer posttraumatischer Schmerz, chronischer neuropathischer Schmerz, chronischer Kopf- und Ge- sichtsschmerz, chronischer viszeraler Schmerz, chronischer muskuloskelettaler Schmerz.

$\mathrm{Zu}$ den einzelnen Schmerzgruppen wird es genauere Definitionen und Querverweise geben. Am Beispiel der chronischen Kopfschmerzen kann dies genauer erläutert werden. So bleiben Kopfschmerzen weiterhin Bestandteil des Kapitels Neurologie im ICD-11. Dabei wird die dritte Ausgabe der Kopfschmerzklassifikation der International Headache Society als Grundlage ge-

nommen. Die chronischen Kopfschmerzarten aber (wie chronische $\mathrm{Mi}$ gräne, chronischer Clusterkopfschmerz etc.) werden auch in dem Kapitel Schmerz aufgelistet. Es steht dem Benutzer des ICD-11 dann frei, welchen Code er für die jeweilige Schmerzerkrankung verwendet.

Momentan werden die genaueren Unterformen und Erläuterungen erstellt. Eine ausführliche Darstellung findet sich in der Publikation von Treede und Kollegen wie auch online unter: http://id.who.int/icd/entity/1581976053. Prof.Dr.Dr. Stefan Evers

\section{Was hilft gegen den Schmerz bei Knie- und Hüftgelenksarthrose?}

Laut einer Netzwerk-Metaanalyse ist bei Knie- und Hüftarthrose die Schmerztherapie mit Paracetamol am wenigsten wirksam.

\begin{abstract}
Z um Vergleich der Wirksamkeit von Schmerzmitteln werden zunehmend Netzwerk-Metaanalysen eingesetzt. Dabei werden die publizierten Vergleiche zwischen einzelnen Substanzen und Placebo in einem virtuellen Netzwerk dargestellt. Eine solche Netzwerk-Metaanalyse wurde jetzt für die Schmerztherapie bei Knie- und Hüftarthrose veröffentlicht. Die Autoren bewerteten die Wirksamkeit der einzelnen Schmerzmittel (NSAR, Coxibe, Paracetamol) dabei auf unterschiedlichen Ebenen. Insgesamt wurden 74 Studien mit mehr als 58.500
\end{abstract}

Patienten untersucht. Als Zielgrößen wurden die Schmerzlinderung und die körperliche Funktionsverbesserung bestimmt. Die Autoren wählten dann eine minimale relevante Effektstärke (z. B. 9 Punkte auf der NAS von 0 bis 100 für die Schmerzlinderung). Zusammenfassend zeigten die folgenden Substanzen eine signifikante Wirksamkeit: Diclofenac $150 \mathrm{mg}$; Etoricoxib 30, 60 und $90 \mathrm{mg}$; Rofecoxib 25 und $50 \mathrm{mg}$. Bei Diclofenac $150 \mathrm{mg}$ und Etoricoxib $60 \mathrm{mg}$ lagen die statistischen Kenngrößen am höchsten, diese Substanzen haben also die größte
Wahrscheinlichkeit, bei den genannten Schmerzsyndromen wirksam zu sein. Am wenigsten wirksam waren Paracetamol und Naproxen in der konventionellen Dosierung. Nur bei 4.000 mg Paracetamol und $1.000 \mathrm{mg}$ Naproxen zeigte sich eine signifikante Überlegenheit gegenüber Placebo. Bei der Verbesserung der körperlichen Funktionsfähigkeit waren Etoricoxib $60 \mathrm{mg}$ und Diclofenac $150 \mathrm{mg}$ am wirksamsten.

\section{Kommentar}

Diese Studie zeigt auf hohem statistischen Niveau eine Wirksamkeit von Diclofenac und Etoricoxib bei arthrosebedingten Hüftund Kniegelenksschmerzen, zusätzlich war auch Rofecoxib (seit längerem nicht mehr erhältlich) wirksam. Paracetamol in normaler Dosierung und Naproxen in eher niedriger Dosierung haben bei beiden Schmerzindikationen keinen Stellenwert. Insge- 